\title{
Screening of methicillin-resistant Staphylococcus aureus nasal colonization among elective surgery patients in referral hospital in Indonesia
}

\author{
Erni J. Nelwan ${ }^{1 *}$, Robert Sinto ${ }^{1}$, Decy Subekti ${ }^{2}$, Randy Adiwinata ${ }^{1}$, Lia Waslia ${ }^{2}$, Tonny Loho ${ }^{3}$, Dodi Safari ${ }^{4}$ \\ and Djoko Widodo ${ }^{1}$
}

\begin{abstract}
Objective: Methicillin-resistant Staphylococcus aureus (MRSA) colonization is associated with serious surgical site infection in high-risk patients. High prevalence of MRSA colonization was reported in many settings, nonetheless local data is required. The purpose of this study is to identify the prevalence and risk factor of MRSA nasal carriage in adult patients in National Referral Hospital in Indonesia before underwent elective surgical procedure.

Results: From 384 patients, 16.9\% patients of them had undergone orthopaedic surgery, 51.3\% had received antibiotics within the previous 3 -month and $41.1 \%$ patients had history of hospitalization within the previous 1 year. Total of $21.6 \%$ patients were on invasive devices for at least $48 \mathrm{~h}$ before the operation; $24.2 \%$ had an open wound; $19.3 \%$ patients were referred from other hospital/ward. Of these patients, solid tumor without metastasis was the most common factor identified by the Charlson index (38.3\%). Nasal colonization of Gram-positive bacteria was detected in $76.8 \%$; $S$. aureus in $15.6 \%$ of patients $(n=60)$. MRSA was identified in three isolates $(0.8 \%)$ by both culture and polymerase chain reaction (PCR) tests. Due to low prevalence of MRSA nasal carriage, this finding supports the recommendation to not routinely apply mupirocin for nasal decolonization on patient planned for surgery in Indonesia.
\end{abstract}

Keywords: Methicillin-resistant Staphylococcus aureus, Nasal colonization, Pre-operative

\section{Introduction}

Surgical site infection (SSI) remains a major contributor of healthcare associated infection (HAI). Based on Centers for Disease Control and Prevention (CDC) prevalence survey in 2011, there were 157,500 SSI cases among all infections occurred in hospitals [1]. SSI was associated with substantial increased of postoperative hospital stay, rates of hospital readmission, functional disability, hospital cost, and mortality rate [2]. Staphylococcus aureus remains the leading cause of SSI, with half of the S. aureus

\footnotetext{
*Correspondence: ejnelwan@yahoo.com

${ }^{1}$ Division of Tropical and Infectious Diseases, Department of Internal Medicine, Faculty of Medicine Universitas Indonesia, Dr. Cipto

Mangunkusumo General Hospital, Jl. Salemba Raya 6, Jakarta 10430, Indonesia

Full list of author information is available at the end of the article
}

were found resistant to methicillin [3, 4]. SSI caused by Methicillin-Resistant S. aureus (MRSA) are increasing in proportion and associates with more devastating outcome $[2,5]$. Numerous studies found that patients with nasal colonization of MRSA were associated with higher risk of SSI [6-8]. Routine decolonization of MRSA with chlorhexidine bathing and mupirocin nares application before surgery is becoming an interesting strategy option to reduce number of SSI, especially in hospital with high rate of MRSA colonization $[6,9,10]$. However, this strategy is not free of consequence. The emergence of chlorhexidine-resistant bacteria and mupirocin resistance are two concerns raised with the wide spread application of this strategy. Active screening followed by selective decolonization is another strategy but with associated with relatively higher cost due to additional diagnostic 
expense [11]. We aimed to investigate the prevalence of MRSA nasal carriage, to determine suitable MRSA decolonization strategy for surgical patients in Indonesia.

\section{Main text \\ Study design and population}

The cross sectional study was conducted in Cipto Mangunkusumo Hospital, Jakarta, Indonesia, between April and September 2015 which was established as a referral hospital for all over the country (260 million inhabitant). Total capacity for inpatient is 900 beds; with approximately 36,000 inpatients from all wards per year and more than 40,000 surgeries per year. To collect data on MRSA colonization among elective surgical patients, we screened all adult patients admitted to the hospital and assigned for an elective surgery. Two hours prior to screening procedure, the list of all adult patients that were planned for elective surgery in the next $24 \mathrm{~h}$ were received. Those who met with the inclusion criteria: aged $\geq 18$ years old, assigned for an elective surgery in the next $24 \mathrm{~h}$, and willing to participate in the study were included consecutively. Patients with active MRSA infection, contraindication for nasal manipulation, and underwent obstetric-gynecology procedure were excluded in this study. Written informed consent was asked before specimens from nasal swab were taken.

\section{Specimen collection}

Nasal swabs were collected using a sterile dry cotton swab from all eligible patients approximately $12 \mathrm{~h}$ before the surgery. Swabs were placed into Stuart Transport Medium and was inoculated onto sheep blood agar plate and mannitol salt agar plate (Oxoid, Hamshire, UK). The culture plates were examined after 24-48 h of incubation at $35^{\circ} \mathrm{C}$. The presumptive $S$. aureus bacteria were than confirmed by catalase and oxidase tests and stored in Tryptone Soya Broth-Glycerol for further analysis [12]. Other bacterial isolates were identified using a standard protocol in the Department of Clinical Pathology, Cipto Mangunkusumo General Hospital, Jakarta.

\section{Antimicrobial susceptibility testing}

Antibiotic susceptibility testing was performed by the disk diffusion method using guidelines established by the Clinical and Laboratory Standards Institute (CLSI) [13]. A total of seven selected antibiotic disks (Oxoid, Hamshire, UK) were used in the test. Those were chloramphenicol $(30 \mu \mathrm{g})$, erythromycin $(15 \mu \mathrm{g})$, tetracycline $(30 \mu \mathrm{g})$, cefoxitin $(30 \mu \mathrm{g})$, oxacillin $(1 \mu \mathrm{g})$, gentamicin $(10 \mu \mathrm{g})$ and trimethoprim-sulfamethoxazole $(25 \mu \mathrm{g})$. CLSI guidelines were used for interpretating the zones of inhibition [13]. The organism use for quality control was S. aureus ATCC 25923.

\section{Detection of mecA and nuc genes}

A few colonies was suspended in TE buffer $(10 \mathrm{mM}$ Tris- $\mathrm{HCl}, 1 \mathrm{mM} \mathrm{Na}{ }_{2} \mathrm{EDTA}, \mathrm{pH}$ 8.0) solution and heated at $100{ }^{\circ} \mathrm{C}$ for $10 \mathrm{~min}$ and instantly frozen at $-20{ }^{\circ} \mathrm{C}$ for $5 \mathrm{~min}$. The lysates were centrifuged at $1000 \times g$ for $10 \mathrm{~min}$. Polymerase chain reaction (PCR) targeting staphylococcal nuclease (nuc) and methicillin-resistance ( $m e c A$ ) genes for detection of $S$. aureus and MRSA isolates were performed as described previously $[12,14,15]$. In short, the reaction mixture contained GoTaq Green Master Mix (Promega), Primers for amplification of nuc genes: $5^{\prime}$-TCAGCAAATGCATCACAAACAG-3' and 5'-CGTAAATGCACTTGCTTCAGG-3' and primers for amplification of $m e c A$ gene: $\quad 5^{\prime}$-GGGATCATAGCGTCATTATTC-3' ${ }^{\prime}$ and $5^{\prime}$-AACGATTGTGACACGATAGCC-3' at $10 \mu \mathrm{M}$ concentration, and $1 \mu \mathrm{l}$ of DNA template. The presence of $255 \mathrm{bp}$ amplicon for $n u c$ and $527 \mathrm{bp}$ amplicon for mecA respectively were detected by electrophoresis on $1.5 \%$ agarose gel and visualized with SYBR safe DNA gel stain (Invitrogen, USA).

\section{Statistical analysis}

All data was processed using Statistical Program for Social Sciences (Version 20; SPSS Inc., Chicago, IL, USA) and results will be presented descriptively.

\section{Results}

Between April and September 2015, a total of 766 patients undergoing various surgeries were screened and 397 patients were eligible for the study. Among them, 384 patients gave written consent to participate in the study (see Fig. 1). The median age of study population was 46 (IQR:25.0) years old and 41.9\% were male. The median length of stay the patients before operation were 2 (IQR:8.0) days. Among 384 patients, 65 (16.9\%) underwent orthopedic surgery, 60 (15.6\%) for digestive surgery, $60(15.6 \%)$ for ear-nose-throat surgery and 58 (15.1\%) for oncology surgery (Table 1).

\section{MRSA risk factor and patient's comorbidities}

The median score of 2 (IQR: 2) from Charlson comorbidity index to determine the risk of MRSA colonization (see Additional file 1). In addition to that, other risk factors such as history of antibiotics usage in the past 3 months (51.3\%), history of hospitalization in the last 12 months (41.1\%) were also identified among this group of patients. More than $20 \%$ of patients had open wound and were on invasive devices for $48 \mathrm{~h}$ prior to the screening; and 19.3\% patient were referred from other hospital or transferred from other wards. Neither history of past colonization and infection of MRSA nor history of living in nursing home were identified. 


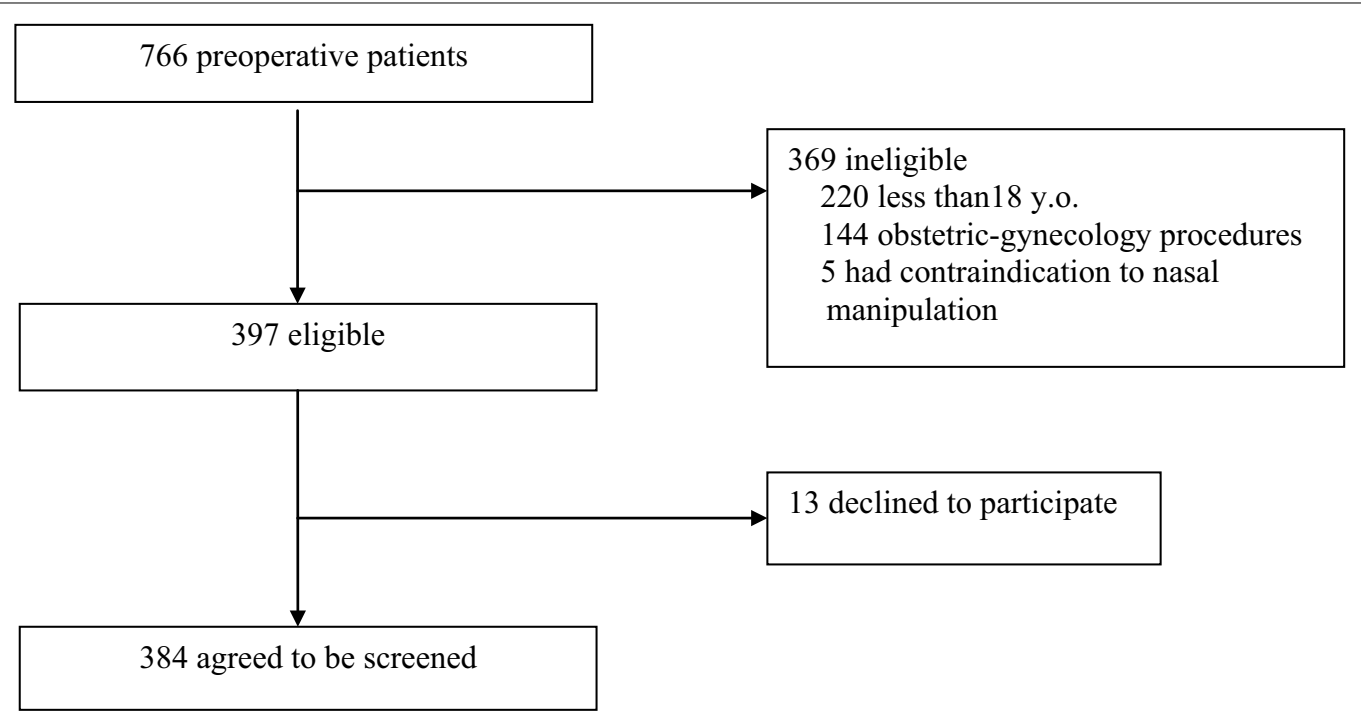

Fig. 1 The flowchart of patients selection

\section{Table 1 Characteristics of participant}

\begin{tabular}{lc}
\hline & N (\%) \\
\hline Male & $161(41.9)$ \\
Age (years old), median (interquartile) & $46(25.0)$ \\
Type of operation & \\
Orthopedics & $65(16.9)$ \\
Digestive & $60(15.6)$ \\
Ear-nose-throat & $60(15.6)$ \\
Oncology & $58(15.1)$ \\
Vascular & $38(9.9)$ \\
Cardiology & $29(7.6)$ \\
Neurology & $28(7.3)$ \\
Oral & $27(7.0)$ \\
Plastic-reconstruction & $9(2.3)$ \\
Urology & $7(1.8)$ \\
Comorbidities & \\
Tumor without metastases & $147(8.3)$ \\
Congestive heart failure & $29(7.6)$ \\
Diabetes with end organ damage & $28(7.3)$ \\
Charlson comorbidity index, median (IQR) & $2(2)$ \\
\hline
\end{tabular}

\section{Staphylococcus aureus and MRSA nasal colonization}

The vast majority of nasal swab cultures results were Gram-positive bacteria (76.5\%). A total of 168 patients (43.8\%) were colonized with Staphylococcus epidermidis and 57 patients (14.8\%) had positive nasal swabs for Methicillin-Sensitive Staphylococcus aureus (MSSA). (Table 2.) Three isolates that showed MRSA were determined by cefoxitin diffusion test and PCR therefore given the prevalence rate of $0.8 \%$ (Table 2).

\section{MRSA positive patients}

The colonization of MRSA was found in three patients planned for orthopaedic, vascular and digestive surgery. These patients were hospitalized in minimum of $1-5$ days before cultures were taken. The Charlson comorbidity index was two for two patients and six for one patient.

\section{Discussion}

During study period, we found a very low prevalence (0.8\%) of MRSA nasal carriage by culture and PCR among preoperative patients compared to report from other countries. One hospital in Singapore found the prevalence of MRSA colonization was 10.6\% [16]. While Hadley, et al. reported prevalence of MRSA colonization anterior nasal was 3.5\% among patients underwent total joint replacement in hospital in United States [17]. Prevalence of $4.25 \%$ was reported in retrospective study among cardiothoracic and neurological surgical patients in United States [18]. A recent study by Santosaningsih, et al. found that MRSA carriage rate was $4.3 \%$ among surgical patients in three academics hospitals in Indonesia. In the study, the screening was performed at the time of discharge and patients that discharged within $48 \mathrm{~h}$ of admission were excluded. Subgroup analysis of three hospitals that participated in this study revealed a significant variation number of MRSA carriage, i.e. 8.0\% in Malang-East Java, 5.9\% in Semarang-Central Java and $0.4 \%$ in Denpasar-Island of Bali [19]. More recent publication from Kuntaman found MRSA prevalence of $3.9 \%$ from nasal swab among patients on admission to medical wards in Surabaya [20]. Difference in prevalence rate between each hospital, reflected the need of local data to 
Table 2 Microbiological culture findings

\begin{tabular}{lc}
\hline Bacteria & N (\%) \\
\hline Methicillin-sensitive Staphylococcus aureus & $57(14.8)$ \\
Methicillin-resistant Staphylococcus aureus & $3(0.8)$ \\
Staphylococcus epidermidis & $168(43.8)$ \\
Staphylococcus saprophyticus & $57(14.8)$ \\
Klebsiella pneumonia & $27(7.0)$ \\
Bacillus sp. & $26(6.8)$ \\
Enterobacter enterogenes & $19(4.9)$ \\
Escherichia coli & $9(2.3)$ \\
Others* & $18(4.8)$ \\
\hline
\end{tabular}

* Alpha-hemolytic Streptococcus sp. (1.8), Acinetobacter baumannii (0.5), Nonhemolytic Streptococcus sp. (0.5), Pseudomonas aeruginosa (0.5), Streptococcus viridans (0.3), Acinetobacter Iwoffii (0.3), Citrobacter freundii (0.3), Enterobacter faecalis (0.3)

assess the needs of routine or selective decolonization protocol in preoperative patients.

The low number of MRSA colonization in our study may be influenced by short duration of hospitalization (median $=2$ days) before the specimens were obtained. This may reflect the low burden of community-acquired MRSA in Indonesia [21]. In addition, no patient had history of MRSA infection and lived in nursing home, which in many studies reported as important risk factor of MRSA colonization [16, 22, 23].

Our subjects had well-known significant independent risk factors of MRSA such as the history of antibiotics usage, invasive medical instrumentation, history of recent hospitalization, open wound, and also mixed cases of medical comorbidities which assessed with Charlson comorbidity index. Some of comorbidities that related with MRSA infection were diabetes, chronic kidney disease, skin infections, pulmonary disease, congestive heart failure, and immunosuppression $[24,25]$.

Low prevalence of MRSA colonization in this study supports the previous study of low MRSA colonization burden in Indonesia $[19,20]$. Therefore, routine decolonization strategy may not be appropriate to be implemented in Indonesia. Routine screening to identify MRSA colonization by culture are not only cost burdening for developing countries, but also time consuming $[26,27]$. While PCR can give rapid result for screening, it is not readily available in all hospitals. Routine decolonization with mupirocin may more cost effective however it may raise the possibility of resistance and lead to treatment failure $[4,5]$. Nowadays, there were increasing trend of mupirocin resistance among MRSA, some of them were caused by extended and excessive use of mupirocin ointment $[28,29]$. Selective swabbing and decolonization for high risk patients may be more appropriate for limited resources countries [26].
To summarize, a low prevalence of MRSA nasal carriage was found among patients performed elective surgery in the national referral hospital in Indonesia. This finding may supports the recommendation to not routinely apply mupirocin for nasal decolonization on patient plan for surgery in Indonesia.

\section{Limitations}

Our study was limited to only one site swabbing for the MRSA colony. Therefore, We might miss the MRSA colony in other areas such as axilla, throat, perineum, and anal [19], although anterior nasal is the highest site of colonization [3]. Another limitation is the one-center study that threatened the external validity to be generalized in Indonesia. Further similar prevalence study should be done across all hospitals in Indonesia, to accurately describe the burden of MRSA colonization among preoperative patients.

\section{Additional file}

Additional file 1. Comorbidities of the participants

\section{Abbreviations}

MRSA: methicillin resistant Staphylococcus aureus; MSSA: methicillin sensitive Staphylococcus aureus; HAl: healthcare associated infection; CDC: Centers for Disease Control and Prevention; PCR: polymerase chain reaction; SBA: sheep blood agar; MSA: mannitol salt agar; CLSI: Clinical and Laboratory Standards Institute; TE: Tris-EDTA

\section{Authors' contributions}

EJN, RS, DW conceived the study. EJN, RS, DW designed and initiated the study. RA worked on data collection and field organization of samples. DS1, DS2, TL, LW responsible for the laboratory analysis. EJN, RS, RA performed statistical analysis of the data. All the authors contributed to writing the manuscript. All authors read and approved the final manuscript.

\section{Author details}

${ }^{1}$ Division of Tropical and Infectious Diseases, Department of Internal Medicine, Faculty of Medicine Universitas Indonesia, Dr. Cipto Mangunkusumo General Hospital, Jl. Salemba Raya 6, Jakarta 10430, Indonesia. ${ }^{2}$ Eijkman Oxford Clinical Reseach Unit, Jl. Diponegoro No. 69, Jakarta 10430, Indonesia. ${ }^{3}$ Department of Clinical Pathology, Faculty of Medicine Universitas Indonesia, Dr. Cipto Mangunkusumo General Hospital, Jl. Salemba Raya 6, Jakarta 10430, Indonesia.

${ }_{4}^{4}$ Eijkman Institute for Molecular Biology, J. Diponegoro No. 69, Jakarta 10430, Indonesia.

\section{Acknowledgements}

Not applicable.

\section{Competing interests}

The authors declare that they have no competing interests.

\section{Availability of data and materials}

The raw data supporting the findings contained within this manuscript will be shared upon request submitted to the corresponding author. Identifying patient data will not be shared.

\section{Consent for publication}

Not applicable. 


\section{Ethics approval and consent to participate}

This study has been reviewed and approved by the ethical committee of the Faculty of Medicine, Universitas Indonesia (Number:82/UN2.F1/ETIK/2015), Jakarta, Indonesia. Written informed consent was obtained from all the patients or guardians prior to enrolment.

\section{Funding}

This study was funded by Operational Research Grant 2015 from Cipto Mangunkusumo Hospital.

\section{Publisher's Note}

Springer Nature remains neutral with regard to jurisdictional claims in published maps and institutional affiliations.

Received: 14 October 2017 Accepted: 9 January 2018

Published online: 22 January 2018

\section{References}

1. Centers for Disease Control and Prevention. HAl data and statistics. In: Healthcare-associated infections. CDC. 2016. http://www.cdc.gov/hai/ surveillance/index.html. Accessed 20 Apr 2016.

2. Reichman DE, Greenberg JA. Reducing surgical site infections: a review. Rev Obstet Gynecol. 2009;2:212-21.

3. Cavalcanti SM, Franca ER, Cabral C, Vilela MA, Montenegro F, Menezes D, et al. Prevalence of Staphylococcus aureus introduced into intensive care units of a university hospital. Braz J Infec Dis. 2005;9:56-63.

4. Anderson MJ, David ML, Scholz M, Bull SJ, Morse D, Hulse-Stevens M, et al. Efficacy of skin and nasal povidone-iodine preparation against mupirocin-resistant methicillin-resistant Staphylococcus aureus and S. aureus within the anterior nares. Antimicrob Agents Chemother. 2015:59:2765-73.

5. Edmiston CE Jr, Ledeboer NA, Buchan BW, Spencer M, Seabrook GR, Leaper D. Is Staphylococcal screening and suppression an effective interventional strategy for reduction of surgical site infection? Surg Infect. 2016;17:158-66.

6. Kim DH, Spencer M, Davidson SM, Li L, Shaw JD, Gulczynski D, et al. Institutional prescreening for detection and eradication of methicillin-resistant Staphylococcus aureus in patients undergoing elective orthopaedic surgery. J Bone Joint Surg Am. 2010;92:1820-6.

7. Kalra L, Camacho F, Whitener CJ, Du P, Miller M, Zalonis C, et al. Risk of methicillin-resistant Staphylococcus aureus surgical site infection in patients with nasal MRSA colonization. Am J Infect Control. 2013;41:1253-7

8. Thakkar V, Ghobrial GM, Maulucci CM, Singhal S, Prasad SK, Harrop JS, et al. Nasal MRSA colonization: impact on surgical site infection following spine surgery. Clin Neurol Neurosurg. 2014;125:94-7.

9. Bebko SP, Green DM, Awad SS. Effect of a preoperative decontamination protocol on surgical site infections in patients undergoing elective orthopedic surgery with hardware implantation. JAMA Surg. 2015;150:390-5.

10. Chen AF, Wessel CB, Rao N. Staphylococcus aureus screening and decolonization in orthopaedic surgery and reduction of surgical site infections. Clin Orthop Relat Res. 2013;471:2383-99.

11. Roth VR, Longpre T, Coyle D, Suh KN, Taljaard M, Muldoon KA, et al. Cost analysis of universal screening vs. risk factor-based screening for methicillin-resistant Staphylococcus aureus (MRSA). PLoS ONE. 2016;11:e0159667.

12. Safari $D$, Harimurti K, Khoeri MM, Waslia L, Mudaliana S, A'yun HQ, et al. Staphylococcus aureus and Streptococcus pneumoniae prevalence among elderly adults in Jakarta, Indonesia. Southeast Asian J Trop Med Public Health. 2015;46:465-71.

13. Clinical and Laboratory Standards Institute (CLSI). M100-S25 Performance standards for antimicrobial susceptibility testing; Twenty-fifth informational supplement. Wayne: Clinical and Laboratory Standards Institute; 2015.

14. Poulsen AB, Skov R, Pallesen LV. Detection of methicillin resistance in coagulase-negative staphylococci and in staphylococci directly from simulated blood cultures using the EVIGENE MRSA Detection Kit. J Antimicrob Chemother. 2003;51:419-21.

15. Liebner S, Harder J, Wagner D. Bacterial diversity and community structure in polygonal tundra soils from Samoylov Island, Lena Delta, Siberia. Int Microbiol. 2008;11:195-202.

16. Oh ML, Tan SY. P054: Prevalence and risk factor analysis for methicillinresistant Staphylococcus aureus colonization in an acute care hospital. Antimicrob Resist Infect Control. 2013;2:P54

17. Hadley S, Immerman I, Hutzler L, Slover J, Bosco J. Staphylococcus aureus decolonization protocol decreases surgical site infections for total joint replacement. Arthritis. 2010;2010:924518.

18. Kapoor R, Barnett CJ, Gutmann RM, Yildiz VO, Joseph NC, Stoicea N, et al. Preoperative prevalence of Staphylococcus aureus in cardiothoracic and neurological surgical patients. Front Public Health. 2014;2:204.

19. Santosaningsih D, Santoso S, Budayanti NS, Kuntaman K, Lestari ES, Farida $\mathrm{H}$, et al. Epidemiology of Staphylococcus aureus harboring the mecA or panton-valentine leukocidin genes in hospitals in Java and Bali, Indonesia. Am J Trop Med Hyg. 2014;90:728-34.

20. Kuntaman K, Hadi U, Setiawan F, Koendori EB, Rusli M, Santosaningsih D, et al. Prevalence of methicillin resistant Staphylococcus aureus from nose and throat of patients on admission to medical wards of DR Soetomo Hospital, Surabaya, Indonesia. Southeast Asian J Trop Med Public Health. 2016:47:66-70

21. David MZ, Daum RS. Community-associated methicillin-resistant Staphylococcus aureus: epidemiology and clinical consequences of an emerging epidemic. Clin Microbiol Rev. 2010;23:616-87.

22. Hidron Al, Kourbatova EV, Halvosa JS, Terrell BJ, McDougal LK, Tenover FC, et al. Risk factors for colonization with methicillin-resistant Staphylococcus aureus (MRSA) in patients admitted to an urban hospital: emergence of community-associated MRSA nasal carriage. Clin Infect Dis. 2005;41:159-66.

23. Lee BY, Bartsch SM, Wong KF, Singh A, Avery TR, Kim DS, et al. The importance of nursing homes in the spread of methicillin-resistant Staphylococcus aureus (MRSA) among hospitals. Med Care. 2013;51:205-15.

24. Huang SS, Hinrichsen VL, Datta R, Spurchise L, Miroshnik I, Nelson K, et al. Methicillin-resistant Staphylococcus aureus infection and hospitalization in high-risk patients in the year following detection. PLOS ONE. 2011;6:e24340.

25. McKinnell JA, Miller LG, Eells SJ, Cui E, Huang SS. A Systematic literature review and meta-analysis of factors associated with MRSA colonization at time of hospital or ICU admission. Infect Control Hosp Epidemiol. 2013:34:1077-86.

26. Kang J, Mandsager P, Biddle AK, Weber DJ. Cost-effectiveness analysis of active surveillance screening for methicillin-resistant Staphylococcus aureus in an academic hospital setting. Infect Control Hosp Epidemiol. 2012;33:477-86.

27. Hubben G, Bootsma M, Luteijn M, Glynn D, Bishai D, Bonten M, et al. Modelling the costs and effects of selective and universal hospital admission screening for methicillin-resistant Staphylococcus aureus. PLoS ONE. 2011;6:e14783.

28. Simor AE, Stuart TL, Louie L, Watt C, Ofner-Agostini M, Gravel D, et al. Mupirocin-resistant, methicillin-resistant Staphylococcus aureus strains in Canadian hospitals. Antimicrob Agents Chemother. 2007:51:3880-6.

29. Vasquez JE, Walker ES, Franzus BW, Overbay BK, Reagan DR, Sarubbi FA. The epidemiology of mupirocin resistance among methicillin-resistant Staphylococcus aureus at a Veterans' Affairs hospital. Infect Control Hosp Epidemiol. 2000;21:459-64. 